Meta

Journal des traducteurs

Translators' Journal

\title{
Aventures et mésaventures de la créativité chez les débutants
}

\section{Georges L. Bastin}

Volume 48, numéro 3, septembre 2003

Traduction et enseignement

Translation and teaching

URI : https://id.erudit.org/iderudit/007595ar

DOI : https://doi.org/10.7202/007595ar

Aller au sommaire du numéro

Éditeur(s)

Les Presses de l'Université de Montréal

ISSN

0026-0452 (imprimé)

1492-1421 (numérique)

Découvrir la revue

Citer cet article

Bastin, G. L. (2003). Aventures et mésaventures de la créativité chez les débutants. Meta, 48(3), 347-360. https://doi.org/10.7202/007595ar

\section{Résumé de l'article}

Dans le cadre du cours d'initiation à la traduction professionnelle à l'Université de Montréal, avec pour fondement la théorie fonctionnelle de Nord (1991, 1997), nous nous sommes donné comme objectif général d’apprentissage l'affranchissement de la littéralité et comme stratégie d'enseignement la stimulation de la créativité. Afin d'évaluer les résultats de cette approche " créative ", nous avons comparé les performances d'une quarantaine d'étudiants en début d'apprentissage et à l'issue de 45 heures d'enseignement. L'analyse d'erreurs a révélé, comme il était somme toute normal de s'y attendre, un nombre de fautes de langue et de sens considérablement moindre dans l'exercice de fin de cours. Toutefois, des fautes d'un nouvel ordre apparaissent comme conséquence de l'application de certains principes théoriques fonctionnalistes. La présente étude examine et illustre les tenants et les aboutissants de l'adoption d'une approche théorique comme la nôtre dans un cours d'initiation à la traduction professionnelle.
Ce document est protégé par la loi sur le droit d'auteur. L'utilisation des services d’Érudit (y compris la reproduction) est assujettie à sa politique d'utilisation que vous pouvez consulter en ligne.

https://apropos.erudit.org/fr/usagers/politique-dutilisation/ 


\title{
Aventures et mésaventures de la créativité chez les débutants
}

\author{
GEORGES L. BASTIN \\ Université de Montréal, Montréal, Canada \\ georges.bastin@umontreal.ca
}

\begin{abstract}
RÉSUMÉ
Dans le cadre du cours d'initiation à la traduction professionnelle à l'Université de Montréal, avec pour fondement la théorie fonctionnelle de Nord (1991, 1997), nous nous sommes donné comme objectif général d'apprentissage l'affranchissement de la littéralité et comme stratégie d'enseignement la stimulation de la créativité. Afin d'évaluer les résultats de cette approche «créative», nous avons comparé les performances d'une quarantaine d'étudiants en début d'apprentissage et à l'issue de 45 heures d'enseignement. L'analyse d'erreurs a révélé, comme il était somme toute normal de s'y attendre, un nombre de fautes de langue et de sens considérablement moindre dans l'exercice de fin de cours. Toutefois, des fautes d'un nouvel ordre apparaissent comme conséquence de l'application de certains principes théoriques fonctionnalistes. La présente étude examine et illustre les tenants et les aboutissants de l'adoption d'une approche théorique comme la nôtre dans un cours d'initiation à la traduction professionnelle.
\end{abstract}

\section{ABSTRACT}

Drawing on Nordian functional theory (1991, 1997), the introductory course in professional translation I give at the Université de Montréal pursues the general objective of helping students break away from literalness while using a teaching strategy which stimulates creativity. To assess the results of this "creative" approach, I compared the performance of 40 students at the beginning of the course with their performance at the end of 45 hours of teaching. Error analysis revealed, predictably, significantly fewer language and meaning errors by the end of the course. However, a new type of error surfaced from the application of certain functionalist theoretical principles. This paper examines the intricacies of adopting such an approach in the teaching of this type of course.

\section{MOTS-CLÉS/KEYWORDS}

créativité, enseignement de la traduction, fonctionnalisme, analyse d'erreurs, initiation

La pédagogie en général, la pédagogie de la traduction en particulier, est un défi constant lancé à la capacité de l'enseignant à définir des objectifs d'apprentissage, conçus selon une expérience attestée, ce qui en garantit la validité "universelle», mais, hélas, toujours inadaptés au groupe d'étudiants «ponctuel» pour qui ils sont élaborés. Rien, en effet, ne permet de prévoir que tel plan de cours connaîtra le même succès d'année en année. Il ne faudrait toutefois pas conclure au caractère frustrant de la démarche, disons plutôt qu'elle réserve souvent des surprises, ce qui, somme toute, en fait tout l'attrait.

Le présent travail tentera de rendre compte de la première étape d'une expérience en cours, menée auprès d'un groupe d'étudiants de traduction en première année universitaire, dans le cadre du cours Méthodologie de la traduction ${ }^{1}$. Cette première 
étape vise à mesurer l'impact de l'approche théorique adoptée sur la performance pratique des étudiants. Nous présenterons d'abord notre approche théorique du cours d'introduction à la traduction professionnelle, puis la méthodologie choisie pour l'expérience; nous examinerons ensuite les résultats de l'étude et en tirerons quelques conclusions.

\section{Approche théorique}

Notre postulat, depuis plusieurs années, et exposé précédemment (Bastin 1999, 2001 et 2002), est simple et complexe à la fois: l'art de la traduction réside essentiellement dans la réexpression. À compréhension égale, le meilleur rédacteur sera invariablement le meilleur traducteur. Notre "philosophie» est l'approche fonctionnaliste (skopos) de la traduction avec des relents de ce que Robinson appelle «the gutts approach». La réexpression, même si nous continuons de croire qu'elle pourra être mise au jour, cernée et décrite un jour, demeure, en effet, avant tout une question de «tripes».

Dans le cadre du lien que, en tant que disciple de la théorie interprétative, nous établissons entre théorie et pratique pédagogique, l'exercice professionnel, la pratique pédagogique présente et surtout nos recherches sur l'adaptation nous ont conduit à remettre en question l'un des postulats de cette théorie, à savoir que, une fois le message original analysé, appréhendé et déverbalisé, sa reformulation est spontanée...! Si tel était le cas, pourquoi un traducteur chevronné, pour qui la langue et le domaine n'ont plus aucun secret, s'arrêterait-il si souvent devant son clavier, parfois pendant de longues minutes, avant d'accoucher d'une phrase? C'est bien d'un accouchement dont il s'agit toujours, "dans la douleur» ou "comme une lettre à la poste»! Non, la réexpression n'est pas spontanée, elle se construit, se planifie, s'élabore, s'hésite, se corrige et se révise pour aboutir. Le processus est laborieux. Ceux qui traduisent le savent.

C'est pourquoi un cours d'initiation à la traduction, un cours de «méthodologie», en plus, cela va de soi, de préparer l'apprenti traducteur à analyser son texte de départ, se doit d'insister sur la réexpression, la reformulation idiomatique, fonctionnelle, donc non littérale, donc créative. C'est là notre philosophie, notre motto, notre skopos: induire l'apprenti à pleinement assumer sa marge de manœuvre, sa tâche de recréation et sa visibilité. D'où l'accent que nous mettons dans notre cours sur l'antilittéralité, l'anti-mécanique, l'anti-automatique au bénéfice de la naturalité, de l'idiomatique et de la recréation. Et cela, à outrance. Avec toute l'exagération qui nous caractérise. Dans quel but? Que les étudiants comprennent, dès le premier jour qu'ils sont des langagiers à part entière, non des répétiteurs; qu'ils sont des communicateurs directs, non des boîtes vocales!

Mais de la même manière que toute médaille a son revers, tout objectif d'apprentissage, ou toute "stratégie d'apprentissage» ou "visée», lorsqu'elle est extrême ou jusqu'à un certain point utopique, comporte sa contrepartie de "désapprentissage». Ou «Not all creativity is good» (Pym 1998: 117). C’est en tout cas ce qu'a révélé l'expérience dont nous voulons rendre compte.

Il convient de préciser maintenant notre concept de créativité. Premièrement, nous emprunterons la définition à Kussmaul: «A creative product must be novel and must contain an element of surprise, it must be singular or at least unusual, but at 
the same time it must, of course, fulfil certain needs and fit in with reality» (cf. Preiser 1976: 2f, cité par Kussmaul 1095: 39).

Ce que Kussmaul (1998: 118) résume par: «the products [...] should be new in one way or another and also [...] they should be adequate to the task». Il s'agit donc de faire du neuf pourvu que ce neuf soit fonctionnel. Pour l'essentiel, la créativité à atteindre chez les apprentis traducteurs est synonyme de résistance à la forme de l'original ou «non littéralité». Vu le texte choisi pour l'expérience, il n'y aura pas, à proprement parler, d'exemples «créatifs» à montrer, seules des «déviations délibérées de la littéralité» pour reprendre l'expression de García Landa (1978). «These translations can be regarded as creative because they are new in the sense that they differ from the source text» (Kussmaul 98: 121).

Un autre aspect essentiel de la créativité est le suivant: «creativity is not a gift of the select few but a basic feature of the human mind» (Kussmaul 1995: 52). En effet, il convient de détruire chez les apprentis traducteurs le mythe d'une créativité qui serait l'apanage des artistes et des génies. Le traducteur est de toute manière toujours contraint par le message source auquel, en principe, il ne touche pas. C'est donc de recréation qu'il s'agit. Le cours d'initiation se doit, à notre avis, d'encourager la réexpression la moins servile possible et les débutants en sont capables. La traduction des titres constitue un excellent moyen de susciter l'imagination. Voici quelques exemples ${ }^{2}$ tirés de copies d'étudiants en début d'apprentissage:

\begin{tabular}{|c|c|}
\hline $\begin{array}{l}\text { 1. Springing Free: Outdoor Activities } \\
\text { to Share with your Toddler } \\
\text { Petits loisirs pour de petits rayons de soleil } \\
\text { Changez d'air, emmenez jouer votre enfant } \\
\text { Après l'hiver, on se libère } \\
\text { Après l'ennui, le printemps } \\
\text { Au printemps, prenez la clé des champs }\end{array}$ & $\begin{array}{l}\text { 2. You can't lead by pushing } \\
\text { La carotte ou le bâton? } \\
\text { Subordination sans frustration } \\
\text { Faut pas pousser! } \\
\text { Leadership: } 1^{\text {re }} \text { leçon } \\
\text { Rien ne sert de pousser, tout vient à point à } \\
\text { qui sait mener } \\
\text { Autocratie n'est pas leadership }\end{array}$ \\
\hline $\begin{array}{l}\text { 3. Animal Farm } \\
\text { Les Américains et leur basse-cour } \\
\text { Le nouveau règne animal } \\
\text { L'animalerie des Américains } \\
\text { La grande ménagerie américaine } \\
\text { L'homme, le meilleur ami des animaux } \\
\text { Animal, rit! } \\
\text { L'animalternité des Américains }\end{array}$ & $\begin{array}{l}\text { 4. The Translator Workstation } \\
\text { Traducteurs, à vos postes! } \\
\text { La traduction à portée de votre souris } \\
\text { Le click du traducteur } \\
\text { Le «traducnateur» }\end{array}$ \\
\hline $\begin{array}{l}\text { 5. Kisses and kibbles } \\
\text { Câlins et gâteries } \\
\text { Bises et biscuits } \\
\text { Becs et biscuits } \\
\text { Bouchées et baisers }\end{array}$ & $\begin{array}{l}\text { 6. Wag and Purr } \\
\text { Ron-ron et patapon! }\end{array}$ \\
\hline $\begin{array}{l}\text { 7. Its time has come } \\
\text { Fêtons tous son avènement! } \\
\text { Il était temps! } \\
\text { Son règne est arrivé }\end{array}$ & \\
\hline
\end{tabular}


La réexpression créative est aussi un processus, difficile à saisir s'il en est... Plusieurs auteurs ont tenté de le décrire. Kussmaul (1995: 39-53) reprend, pour ce faire, le modèle psychologique en quatre phases: preparation, incubation, illumination et evaluation. Et suggère trois techniques de stimulation de la créativité: le remueméninges (brainstorming) qui encourage tous les types d'associations et diffère tout jugement; la technique des activités parallèles (parallel-activity technique) qui consiste à «oxygéner» l'esprit pour un moment; et la production divergente (divergent production), inspirée de la pensée divergente (divergent thinking) de Guilford, qui vise à la production d'alternatives logiques. En 1999, il penche pour la «lateral thinking» (Brodbeck 1995; De Bono 1990) qui fait ressortir deux éléments essentiels de la pensée créative: le point de vue (perspective) et le centre d'intérêt (focus). Kussmaul se tourne ensuite vers Lakoff et Fillmore pour présenter quatre types de processus mentaux: moving frame to frame, frame to scene, scene to scene et scene to frame, et cinq types de traductions créatives: chaining of categories, picking out scene elements, enlarging a scene, framing a scene and thinking up a new frame ${ }^{3}$.

On le voit, s'attaquer à la créativité, en traduction ou ailleurs, n'est pas chose aisée. Il y a place pour beaucoup de «flou artistique»... Devant des débutants toutefois, il importe de ne pas compliquer les concepts qui sont déjà assez déroutants pour l'étudiant bilingue ou presque qui n'a jamais réfléchi à la complexité du mécanisme naturel qu'il met en œuvre quotidiennement depuis plusieurs années. Ce qu'il faut éviter à tout prix, c'est la paresse intellectuelle qui consiste à comprendre à demi-mot et à s'exprimer à demi-phrase! Si l'expression du traducteur doit s'éloigner de l'original pour être créative (c'est l'essentiel de notre travail), il ne faudrait toutefois pas négliger la compréhension qui, elle aussi, peut être créative (Mackenzie 1998: 202) par l'analyse et la «relecture». Ce que nous visons, c'est en somme que l'étudiant apprenne à s'approprier un texte pour en créer un nouveau.

\section{Méthodologie}

Avec une certaine humilité et beaucoup d'appréhensions, nous nous sommes demandés si, au-delà d'une note moyenne quantitative, nos efforts atteignaient un quelconque résultat qualitatif. C'est ainsi que nous avons décidé de donner à nos étudiants de première année, le $1^{\text {er }}$ jour de cours, alors qu'ils n'ont, en principe, encore eu aucun contact avec la traduction professionnelle, un court texte à traduire. Ce même texte, nous le leur avons redonné à traduire, sans avis préalable, 15 semaines plus tard, au terme du cours. Inutile de dire que le souvenir qu'il en avaient était des plus lointains. Le texte est un extrait du numéro d'avril de World Trade, un magazine économique en ligne non spécialisé. Le texte porte sur l'importance de la traduction dans les milieux d'affaires internationaux:

The rapid globalisation of trade and industry has yet to create a genuine lingua franca - a truly universal language in wich the business of commerce can be conducted. But the need for business and trade partners to communicate clearly across language barriers has never been greater.

Accurate and precise translations of contracts and agreements, technical manuals, memos, letters and reports, for exemple, are needed if global businesses are to operate efficiently. But where do you find professional translators? And what special qualifications do they need? How can you be sure that they're doing the job properly? 
The following article offers some tips on how to go about this often-overlooked, but vital, aspect of international business.

World Trade, April 2000

37 étudiants ont pris part au premier test ( $1^{\text {re }}$ semaine de cours) et 28 ont présenté le second ( $15^{\mathrm{e}}$ semaine). Un total de 25 étudiants ont effectué les deux tests, soit 50 traductions qui ont constitué notre corpus ${ }^{4}$.

La typologie d'erreurs adoptée pour cette étude est classique. Les erreurs ont été regroupées en trois catégories: fautes de langue ou de maitrise inadéquate de la langue d'arrivée (lexique, caractère idiomatique, grammaire, temps verbal, orthographe), fautes de compréhension ou mauvaise analyse du texte de départ (faux sens, contresens, non-sens) et fautes de technique (omission, surtraduction, sous-traduction). La comparaison a comporté plusieurs étapes.

Nous présenterons tout d'abord un relevé systématique des fautes de chacun des tests (Tableau ${ }^{\circ} 1$ et Tableau $n^{\circ} 2$ ). Ensuite, un relevé comparatif du nombre total de fautes (Tableau $\mathrm{n}^{\circ} 3$ ). Puis, un relevé systématique des fautes du premier test qui se répètent dans le second et de celles qui y ont été corrigées (Tableau ${ }^{\circ} 5$ et Tableau ${ }^{\circ}$ 4). Le relevé suivant est naturellement celui des fautes nouvelles commises dans le deuxième test (Tableau ${ }^{\circ} 6$ ). Le dernier tableau récapitule les fautes commises dans les deux tests afin d'en apprécier l'évolution (Tableau ${ }^{\circ} 7$ ).

\section{Discussion des résultats}

Notons tout d'abord un paradoxe assez flagrant de notre étude. Elle est en effet une analyse d'erreurs, alors que l'examen des mécanismes de créativité dans l'expression devrait être fondé sur les réussites, les trouvailles, et non sur les erreurs! Mais notre objectif étant d'évaluer les résultats de notre démarche pédagogique, l'analyse d'erreurs a constitué un instrument utile.

Après avoir corrigé ces tests, nous avons donc entrepris de comparer les erreurs commises dans les tests du premier et du dernier jour. Qu'attendions-nous d'une telle confrontation? L'évidence que nos étudiants étaient moins littéraux, plus idiomatiques et plus créatifs après 15 semaines, le sacre du professeur, quoi!

Pour voir ce qui ressort de la comparaison, examinons les données.

Tableau $n^{\circ} 1$ : Total des fautes Version 1

On observe tout d'abord un nette prépondérance des "fautes de langue» (148 sur un total de 234), dénotant le manque de conscience langagière des débutants. La catégorie «fautes de technique» ne révèle que très peu de fautes (21 sur 234). En fait cette catégorie, en toute rigueur, ne devrait pas exister pour le premier test, puisque les étudiants, qui en sont à leur premier cours, ne possèdent évidemment aucune technique de traduction. La catégorie est ici présente d'abord aux fins de la comparaison, ensuite parce qu'elle permet de relever des fautes qui n'appartiennent ni à la langue ni à la compréhension. Autre constatation en marge: le nombre relativement élevé de fautes (près de dix par étudiant en moyenne) pour un texte aussi court et aussi «inoffensif» montre à quel point le bilinguisme est loin d'être une garantie pour la traduction professionnelle. 
Tableaux $n^{\text {os }} 2$ et 3: Total des fautes Version 2 - Total des fautes V. 1 et V. 2 Ce qui saute aux yeux est d'abord la réduction considérable du total des fautes qui passe de 234 à 159 . Cette réduction vaut pour les fautes de langue (de $63 \%$ à $50 \%$ ) et celles de compréhension (de $27 \%$ à 23,8\%). En revanche l'augmentation des fautes de technique est notable (près de $300 \%$ ), de 21 sur 234 à 41 sur 159, soit de 8,9\% à $25,7 \%$. Ce qui revient à dire qu'après 15 semaines de cours de méthodologie de la traduction, les étudiants commettent davantage de fautes de technique. Nous reviendrons plus loin sur ce paradoxe.

Tableaux $n^{\text {os }} 4$ et 5 : Fautes corrigées - Fautes qui se répètent

Il est très réconfortant pour l'enseignant de constater que sur 234 fautes dans le premier test 208 ont été corrigées, soit 88,8\%, en particulier pratiquement toutes les fautes de langue de la $1^{\text {ère }}$ version (148) sont corrigées (138).

Tableaux $n^{\circ}$ 6: Fautes nouvelles

Il apparaît un total de 133 nouvelles fautes: 70 nouvelles fautes de langue, 25 de compréhension et 38 de technique. D'une part, les fautes de langue (34 de lexique et 27 de grammaire) font état d'une maîtrise encore insuffisante de la langue d'arrivée après le cours de méthodologie. Lorsque l'on sait que la plupart des étudiants ont aussi suivi un cours de rédaction et d'interférence linguistique, ce chiffre est alarmant et appelle des correctifs dans le cursus ou dans les exigences des cours concernés.

D'autre part, si les 21 fautes de technique de la $1^{\text {re }}$ version (rappelons-nous les réserves émises!) sont pratiquement toutes corrigées (18), ce qui confirme qu'il ne s'agissait pas à proprement parler de fautes de technique (appelons-les des fautes d'inexpérience), le nombre de nouvelles fautes est significatif: 38 . Ces fautes peuvent être considérées, après 15 semaines de cours, de «vraies» fautes de technique.

Tableau $n^{\circ}$ 7: Résultats généraux

Ce tableau pourrait entraîner de nombreux commentaires, tous utiles à une meilleure conception et planification d'un cours d'initiation. Dans le cadre de cette étude, le plus important est sans doute la quantité de nouvelles fautes de technique commises (38) et la nature de celles-ci (essentiellement des surtraductions) qui montrent bien le désapprentissage dont nous parlions et les dangers d'une approche comme la nôtre.

\section{Conclusions}

L’analyse des données présentées ci-dessus révèle plusieurs aspects intéressants de l'interaction «théorie-pratique pédagogique»:

1) La comparaison des tableaux 1 et 2, soit le tableau 3, fait apparaître un "progrès » de la part des étudiants qui, globalement, commettent moins de fautes à la fin du cours (réduction considérable des fautes de langue et de compréhension, et donc du total des fautes qui passe de 234 à 159). Le tableau 4 montre en effet que les étudiants ont appris, puisqu'ils ne commettent plus les fautes de la première heure. Nous faisons l'hypothèse qu'un enseignant qui suivrait une approche «littéraliste» obtiendrait vraisemblablement à peu près les mêmes résultats. Il y a peut-être là matière à un projet de recherche conjoint...

2) Par contre, le tableau 3 révèle une nette augmentation (300\%) des fautes de technique. Comment expliquer ce paradoxe? Où est la «méthodologie»? C’est là que résident les 
«mésaventures» de la créativité! D'abord les surtraductions : l'étudiant pèche par excès de fonctionnalisme et sent le besoin de «mâcher la besogne» au lecteur. La faute de méthode est ici une sous-estimation des connaissances du destinataire. Ensuite, les omissions : l'étudiant applique à outrance le principe, cher à son professeur, de «nonimbécillité du lecteur», ce qui revient souvent à une surestimation du savoir partagé par le destinateur. Les fautes de technique semblent donc résulter, pour le groupe d'étudiants concernés et l'approche théorique adoptée, d'une double démarche problématique: l'évaluation adéquate des skopos de départ et d'arrivée.

3) L'apparition de nouvelles fautes de langue s'explique, d'une façon classique, par un maîtrise insuffisante de la langue d'arrivée, mais, dans notre optique, elle est peut-être attribuable à la concentration nécessaire à une «nouvelle» formulation, éloignée de l'original, et donc à un manque de révision.

Comme nous le disions au début, le présent travail rend compte de la première étape d'une recherche sur la pédagogie de la réexpression chez les apprentis traducteurs. Nous l'avons vu, cette première étape s'est cantonnée dans une analyse d'erreurs, ce qui, nous l'avons dit plus haut, constitue un paradoxe. La deuxième consistera précisément à effectuer une «analyse de succès ».

En fin de compte, cette remise en question de notre approche théorique, malgré les mésaventures qu'elle a révélées, ne laisse pas de regret. Notre approche a porté fruit; cette étude nous a montré qu'il fallait cependant modérer quelque peu le culte de la créativité.

\section{NOTES}

1. Expérience menée à l'Université de Montréal à l'automne 2001.

2. Les exemples 2, 3 et 4 sont extraits de textes proposés par Delisle (1993) dans La traduction raisonnée.

3. La discussion de ces diverses descriptions du processus de la réexpression créative dépasse le cadre du présent travail.

4. L'auteur tient à remercier son étudiant de doctorat, M. Miguel Betancourt, pour la saisie, la compilation et la classification minutieuses des données de l'expérience sur laquelle se fonde le présent travail.

\section{RÉFÉRENCES}

BAstin, G. (1997) : «Adaptation» in M. BAKER (réd.), Routledge Encyclopedia of Translation Studies, London, Routledge, p. 5-8.

Bastin, G. (1998) : ¿Traducir o adaptar? Caracas, Universidad Central de Venezuela, DCH/FHE, $208 \mathrm{p}$.

BAstin, G. (1999) : «Comprendre, la belle affaire... mais redire!», Mons, XV Congrès mondial de la FIT Traduction -Transition, p. 48-54.

Bastin, G. (2001): «Evaluating Beginners' Re-Expression and Creativity: A Positive Approach », The Translator, vol. 6, $\mathrm{n}^{\circ} 2$, p. 231-245.

BAstin, G. (2002) : «La traduction, activité onomasiologique», in G. Mareschal et al. (dir.), La formation à la traduction professionnelle, Ottawa, Les Presses de l'Université d'Ottawa, (sous presse).

Brodbeck, K. H. (1995) : Entscheidung zur Kreativität, Darmstadt, Wissenschafliche Buchgesellschaft.

De Bono, E. (1970): Lateral Thinking. A Textbook of Creativity, London, Ward Lock Educational. Delisle, J. (1993): La traduction raisonnée, Ottawa, Les Presses de l'Université d'Ottawa.

Garcia Landa, M. (1978): Les déviations délibérées de la littéralité. Thèse de doctorat, ESIT, Université Paris III, non publiée.

Kussmaul, P. (1995): Training the Translator, Amsterdam/Philadelphia, John Benjamins. 
Kussmaul, P. (1998): «Types of Creative Translating» in A. Chesterman, N. Gallardo San Salvador and Y. Gambier, Translation in Context, Amsterdam/Philadelphia, John Benjamins, p. 117-126.

Mackenzie, R. (1998) : «Creative Problem-Solving and Translator Training», Translators' Strategies and Creativity, Amsterdam/Philadelphia, John Benjamins, p. 201-206.

Nord, C. (1991): Text Analysis in Translation, Trad. C. Nord et P. Sparrow, Amsterdam/Atlanta, Rodopi.

Nord, C. (1997): Translation as a Purposeful Activity. Functionalist Approaches Explained. Manchester, St. Jerome Publishing.

Pyм, A. (1998): «Lives of Henri Albert, Nietzschean Translator» in Translators' Strategies and Creativity, Amsterdam/Philadelphia, John Benjamins, p. 117-125

Robinson, D. (1991): The Translator's Turn, Baltimore, Johns Hopkins University Press.

TABLEAU $\mathrm{N}^{\circ} 1$

Aventures et mésaventures de la créativité chez les apprentis traducteurs

TOTAL FAUTES VERSION $\mathbf{n}^{\circ} \mathbf{1}$

\begin{tabular}{|l|c|c|c|c|c|c|c|c|c|c|c|c|}
\hline Élève & \multicolumn{5}{|c|}{ Fautes de langue } & \multicolumn{3}{c|}{$\begin{array}{c}\text { Fautes de } \\
\text { compréhension }\end{array}$} & \multicolumn{3}{|c|}{$\begin{array}{c}\text { Fautes de } \\
\text { technique }\end{array}$} & $\begin{array}{c}\text { Total } \\
\text { Fautes }\end{array}$ \\
\hline$N^{\text {o }}$ & Lex. & C.i. & Gram. & T.v. & Orth. & F.s. & N.s. & C.s. & Om. & Surt. & Soust. & \\
\hline 1 & & 1 & 7 & & & 2 & 3 & & 2 & & & 15 \\
\hline 2 & 3 & & 2 & & & 2 & & & & & & 7 \\
\hline 3 & 6 & & & & 1 & 3 & & & & & & 10 \\
\hline 5 & 3 & & & & 1 & & & & 1 & & & 5 \\
\hline 6 & & & 4 & & 1 & 1 & & & & & & 6 \\
\hline 8 & 4 & & & & & & & & 2 & & & 6 \\
\hline 9 & 5 & & & & & & 1 & & 1 & & & 7 \\
\hline 10 & 1 & & 4 & & 1 & 1 & & & 1 & & & 8 \\
\hline 12 & 1 & & & & 1 & & 2 & & & & & 4 \\
\hline 13 & 3 & & & & 1 & & 3 & & 1 & & & 8 \\
\hline 14 & 1 & & 1 & & & & 5 & & & & & 7 \\
\hline 15 & 2 & & 1 & & 2 & 4 & & & 3 & & & 12 \\
\hline 16 & 4 & & & & 1 & 4 & 1 & & & 1 & & 11 \\
\hline 17 & 4 & & 1 & & 2 & & & & & 1 & & 8 \\
\hline 18 & 3 & & 1 & & 2 & 2 & 2 & & & & & 10 \\
\hline 19 & 4 & & 6 & & 3 & 1 & 2 & & & & & 16 \\
\hline 22 & 1 & & 2 & & 1 & 1 & 2 & & 1 & & & 8 \\
\hline 23 & 3 & & 1 & & 3 & 2 & & & & & & 9 \\
\hline 25 & 5 & & & & 6 & 1 & & & & & & 12 \\
\hline 26 & 4 & & 2 & & 2 & 1 & 1 & & & 1 & & 11 \\
\hline 28 & 6 & & 3 & & 2 & 2 & & & & & & 13 \\
\hline 30 & & & 1 & 1 & & 3 & & & 2 & & & 7 \\
\hline 31 & 1 & & 2 & & 4 & 3 & 1 & 1 & 3 & & & 15 \\
\hline 33 & 3 & & 3 & & 1 & 7 & 1 & & 1 & & & 16 \\
\hline 37 & 2 & & 1 & & & & & & & & & 3 \\
\hline Total & 69 & 1 & 42 & 1 & 35 & 40 & 24 & 1 & 18 & 3 & 0 & 234 \\
\hline 25 & & & 148 & & & & 65 & & & 21 & \\
\hline
\end{tabular}


TABLEAU $\mathrm{N}^{\circ} 2$

Aventures et mésaventures de la créativité chez les apprentis traducteurs

TOTAL FAUTES VERSION $\mathbf{n}^{\circ} 2$

\begin{tabular}{|l|c|c|c|c|c|c|c|c|c|c|c|c|}
\hline Élève & \multicolumn{5}{|c|}{ Fautes de langue } & \multicolumn{3}{c|}{$\begin{array}{c}\text { Fautes de } \\
\text { compréhension }\end{array}$} & \multicolumn{3}{|c|}{$\begin{array}{c}\text { Fautes de } \\
\text { technique }\end{array}$} & $\begin{array}{c}\text { Total } \\
\text { Fautes }\end{array}$ \\
\hline $\mathrm{N}^{\text {0 }}$ & Lex. & C.i. & Gram. & T.v. & Orth. & F.s. & N.s. & C.s. & Om. & Surt. & Soust. & \\
\hline 1 & 2 & & 3 & & & & 3 & & & 1 & & 9 \\
\hline 2 & 1 & & 1 & & & 1 & & & 1 & & & 4 \\
\hline 3 & 3 & & & 1 & 1 & 2 & & & & 1 & & 8 \\
\hline 5 & 2 & & & & & & & & & 1 & & 3 \\
\hline 6 & 1 & & 2 & & 1 & 1 & & & & 1 & & 6 \\
\hline 8 & 1 & & & & & & & & & & & 1 \\
\hline 9 & 1 & & 1 & & & & 2 & & 1 & & & 5 \\
\hline 10 & & & 1 & & & & & & & & & 1 \\
\hline 12 & 1 & & 2 & & 1 & & 1 & & & 3 & & 8 \\
\hline 13 & 1 & & 1 & & & 1 & & & & & & 3 \\
\hline 14 & 2 & & 1 & & & 3 & & & 2 & & & 8 \\
\hline 15 & 3 & & & & & 1 & & & 2 & 2 & & 8 \\
\hline 16 & 3 & & 1 & & & 1 & & & 1 & 4 & & 10 \\
\hline 17 & 2 & & & & 1 & & & & & & & 3 \\
\hline 18 & 2 & & 3 & & & 1 & & & & 1 & & 7 \\
\hline 19 & 2 & & 2 & & & 3 & & & & 2 & & 9 \\
\hline 22 & & & & & & 2 & & & 1 & 1 & & 4 \\
\hline 23 & 2 & & 1 & & & 1 & & & 1 & & & 5 \\
\hline 25 & 3 & & 2 & & 1 & 1 & 1 & & & 1 & & 9 \\
\hline 26 & 2 & & & 1 & & 1 & & & & 1 & & 5 \\
\hline 28 & 2 & & 2 & & & & 2 & 1 & 1 & 1 & & 9 \\
\hline 30 & 1 & & & 1 & & 1 & & & & 1 & 1 & 5 \\
\hline 31 & & & 2 & & 1 & 2 & & 2 & 1 & 4 & & 12 \\
\hline 33 & 2 & & 4 & & 1 & 3 & & & & 2 & & 12 \\
\hline 37 & 1 & & 1 & & & 1 & & & 1 & 1 & & 5 \\
\hline Total & 40 & 0 & 30 & 3 & 7 & 26 & 9 & 3 & 10 & 30 & 1 & 159 \\
\hline 25 & & & 80 & & & & 38 & & & 41 & \\
\hline
\end{tabular}


TABLEAU $\mathrm{N}^{\circ} 3$

Aventures et mésaventures de la créativité chez les apprentis traducteurs

TOTAL FAUTES V. 1 et V. 2

\begin{tabular}{|c|c|c|c|c|c|c|}
\hline \multirow{2}{*}{$\begin{array}{l}\text { Élève } \\
\mathrm{N}^{\mathrm{o}}\end{array}$} & \multicolumn{2}{|c|}{$\begin{array}{l}\text { Fautes de } \\
\text { langue }\end{array}$} & \multicolumn{2}{|c|}{$\begin{array}{c}\text { Fautes de } \\
\text { compréhension }\end{array}$} & \multicolumn{2}{|c|}{$\begin{array}{l}\text { Fautes de } \\
\text { technique }\end{array}$} \\
\hline & V.1 & V.2 & V.1 & V.2 & V.1 & V.2 \\
\hline 1 & 8 & 5 & 5 & 3 & 2 & 1 \\
\hline 2 & 5 & 2 & 2 & 1 & 0 & 1 \\
\hline 3 & 7 & 5 & 3 & 2 & 0 & 1 \\
\hline 5 & 4 & 2 & 0 & 0 & 1 & 1 \\
\hline 6 & 5 & 4 & 1 & 1 & 0 & 1 \\
\hline 8 & 4 & 1 & 0 & 0 & 2 & 0 \\
\hline 9 & 5 & 2 & 1 & 2 & 1 & 1 \\
\hline 10 & 6 & 1 & 1 & 0 & 1 & 0 \\
\hline 12 & 2 & 4 & 2 & 1 & 0 & 1 \\
\hline 13 & 4 & 2 & 3 & 1 & 1 & 1 \\
\hline 14 & 2 & 3 & 5 & 3 & 0 & 2 \\
\hline 15 & 5 & 3 & 4 & 1 & 3 & 4 \\
\hline 16 & 5 & 4 & 5 & 1 & 1 & 5 \\
\hline 17 & 7 & 3 & 0 & 0 & 1 & 0 \\
\hline 18 & 6 & 5 & 4 & 1 & 0 & 1 \\
\hline 19 & 13 & 4 & 3 & 3 & 3 & 2 \\
\hline 22 & 4 & 0 & 3 & 2 & 1 & 2 \\
\hline 23 & 7 & 3 & 2 & 1 & 0 & 1 \\
\hline 25 & 11 & 6 & 1 & 2 & 0 & 1 \\
\hline 26 & 8 & 3 & 2 & 1 & 1 & 1 \\
\hline 28 & 11 & 4 & 2 & 3 & 0 & 2 \\
\hline 30 & 2 & 2 & 3 & 1 & 2 & 2 \\
\hline 31 & 7 & 3 & 5 & 4 & 3 & 5 \\
\hline 33 & 7 & 7 & 8 & 3 & 1 & 2 \\
\hline 37 & 3 & 2 & 0 & 1 & 0 & 2 \\
\hline $\begin{array}{c}\text { Total } \\
25 \\
\end{array}$ & 148 & 80 & 65 & 38 & 21 & 41 \\
\hline
\end{tabular}

Total fautes version $n^{\circ} 1=234$

Total fautes version $\mathrm{n}^{\circ} 2=159$ 
TABLEAU $\mathrm{N}^{\circ} 4$

Aventures et mésaventures de la créativité chez les apprentis traducteurs

FAUTES CORRIGÉES

\begin{tabular}{|l|c|c|c|c|c|c|c|c|c|c|c|c|}
\hline Élève & \multicolumn{5}{|c|}{ Fautes de langue } & \multicolumn{3}{c|}{$\begin{array}{c}\text { Fautes de } \\
\text { compréhension }\end{array}$} & \multicolumn{3}{|c|}{$\begin{array}{c}\text { Fautes de } \\
\text { technique }\end{array}$} & $\begin{array}{c}\text { Total } \\
\text { Fautes }\end{array}$ \\
\hline $\mathrm{N}^{\text {0 }}$ & Lex. & C.i. & Gram. & T.v. & Orth. & F.s. & N.s. & C.s. & Om. & Surt. & Soust. & \\
\hline 1 & & 1 & 7 & & & 2 & 3 & & 2 & & & 15 \\
\hline 2 & 3 & & 2 & & & 1 & & & & & & 6 \\
\hline 3 & 6 & & & & 1 & 2 & & & & & & 9 \\
\hline 5 & 2 & & & & 1 & & & & 1 & & & 4 \\
\hline 6 & & & 4 & & 1 & & & & & & & 5 \\
\hline 8 & 3 & & & & & & & & 2 & & & 5 \\
\hline 9 & 5 & & & & & & 1 & & 1 & & & 7 \\
\hline 10 & 1 & & 4 & & 1 & 1 & & & 1 & & & 8 \\
\hline 12 & 1 & & & & 1 & & 2 & & & & & 4 \\
\hline 13 & 3 & & & & 1 & & 3 & & 1 & & & 8 \\
\hline 14 & 1 & & & & & & 5 & & & & & 6 \\
\hline 15 & 1 & & 1 & & 2 & 3 & & & 2 & & & 9 \\
\hline 16 & 3 & & & & 1 & 3 & 1 & & & 1 & & 9 \\
\hline 17 & 4 & & 1 & & 1 & & & & & 1 & & 7 \\
\hline 18 & 2 & & & & 2 & 1 & 2 & & & & & 7 \\
\hline 19 & 3 & & 6 & & 3 & & 2 & & & & & 14 \\
\hline 22 & 1 & & 2 & & 1 & & 2 & & & & & 6 \\
\hline 23 & 3 & & 1 & & 3 & 2 & & & & & & 9 \\
\hline 25 & 5 & & & & 6 & 1 & & & & & & 12 \\
\hline 26 & 4 & & 2 & & 2 & 1 & 1 & & & 1 & & 11 \\
\hline 28 & 6 & & 3 & & 2 & 2 & & & & & & 13 \\
\hline 30 & & & 1 & 1 & & 3 & & & 2 & & & 7 \\
\hline 31 & 1 & & 2 & & 4 & 1 & 1 & 1 & 2 & & & 12 \\
\hline 33 & 3 & & 2 & & 1 & 4 & 1 & & 1 & & & 12 \\
\hline 37 & 2 & & 1 & & & & & & & & & 3 \\
\hline Total & 63 & 1 & 39 & 1 & 34 & 27 & 24 & 1 & 15 & 3 & & 208 \\
\hline 25 & & & 138 & & & & 52 & & 18 & \\
\hline
\end{tabular}


358 Meta, XLVIII, 3, 2003

TABLEAU $\mathrm{N}^{\circ} 5$

Aventures et mésaventures de la créativité chez les apprentis traducteurs

FAUTES QUI SE RÉPÈTENT

\begin{tabular}{|c|c|c|c|c|c|c|c|c|c|c|c|c|}
\hline \multirow{2}{*}{$\begin{array}{l}\text { Élève } \\
\mathrm{N}^{\circ} \\
\end{array}$} & \multicolumn{5}{|c|}{ Fautes de langue } & \multicolumn{3}{|c|}{$\begin{array}{c}\text { Fautes de } \\
\text { compréhension }\end{array}$} & \multicolumn{3}{|c|}{$\begin{array}{l}\text { Fautes de } \\
\text { technique }\end{array}$} & \multirow[t]{2}{*}{$\begin{array}{r}\text { Total } \\
\text { Fautes }\end{array}$} \\
\hline & Lex. & C.i. & Gram. & T.v. & Orth. & F.s. & N.s. & C.s. & Om. & Surt. & Soust. & \\
\hline 1 & & & & & & & & & & & & 0 \\
\hline 2 & & & & & & & & & & & & 0 \\
\hline 3 & & & & & & 1 & & & & & & 1 \\
\hline 5 & 1 & & & & & & & & & & & 1 \\
\hline 6 & & & & & & 1 & & & & & & 1 \\
\hline 8 & 1 & & & & & & & & & & & 1 \\
\hline 9 & & & & & & & & & & & & 0 \\
\hline 10 & & & & & & & & & & & & 0 \\
\hline 12 & & & & & & & & & & & & 0 \\
\hline 13 & & & & & & & & & & & & 0 \\
\hline 14 & & & 1 & & & 1 & & & & & & 2 \\
\hline 15 & 1 & & & & & 1 & & & 1 & & & 3 \\
\hline 16 & 1 & & & & & 1 & & & & & & 2 \\
\hline 17 & & & & & 1 & & & & & & & 1 \\
\hline 18 & 1 & & 1 & & & 1 & & & & & & 3 \\
\hline 19 & 1 & & & & & 1 & & & & & & 2 \\
\hline 22 & & & & & & 1 & & & 1 & & & 2 \\
\hline 23 & & & & & & & & & & & & 0 \\
\hline 25 & & & & & & & & & & & & 0 \\
\hline 26 & & & & & & & & & & & & 0 \\
\hline 28 & & & & & & & & & & & & 0 \\
\hline 30 & & & & & & & & & & & & 0 \\
\hline 31 & & & & & & 2 & & & 1 & & & 3 \\
\hline 33 & & & 1 & & & 3 & & & & & & 4 \\
\hline 37 & & & & & & & & & & & & 0 \\
\hline \multirow{2}{*}{$\begin{array}{l}\text { Total } \\
25 \\
\end{array}$} & 6 & & 3 & & 1 & 13 & & & 3 & & & \multirow[t]{2}{*}{26} \\
\hline & & & 10 & & & & 13 & & & 3 & & \\
\hline
\end{tabular}


TABLEAU $N^{\circ} 6$

Aventures et mésaventures de la créativité chez les apprentis traducteurs

FAUTES NOUVELLES

\begin{tabular}{|l|c|c|c|c|c|c|c|c|c|c|c|c|}
\hline Élève & \multicolumn{5}{|c|}{ Fautes de langue } & \multicolumn{3}{c|}{$\begin{array}{c}\text { Fautes de } \\
\text { compréhension }\end{array}$} & \multicolumn{3}{c|}{$\begin{array}{c}\text { Fautes de } \\
\text { technique }\end{array}$} & $\begin{array}{c}\text { Total } \\
\text { Fautes }\end{array}$ \\
\hline $\mathrm{N}^{\text {o }}$ & Lex. & C.i. & Gram. & T.v. & Orth. & F.s. & N.s. & C.s. & Om. & Surt. & Soust. & \\
\hline 1 & 2 & & 3 & & & & 3 & & & 1 & & 9 \\
\hline 2 & 1 & & 1 & & & 1 & & & 1 & & & 4 \\
\hline 3 & 3 & & & 1 & 1 & 1 & & & & 1 & & 7 \\
\hline 5 & 1 & & & & & & & & & 1 & & 2 \\
\hline 6 & 1 & & 2 & & 1 & & & & & 1 & & 5 \\
\hline 8 & & & & & & & & & & & & 0 \\
\hline 9 & 1 & & 1 & & & & 2 & & 1 & & & 5 \\
\hline 10 & & & 1 & & & & & & & & & 1 \\
\hline 12 & 1 & & 2 & & 1 & & 1 & & & 3 & & 8 \\
\hline 13 & 1 & & 1 & & & 1 & & & & & & 3 \\
\hline 14 & 2 & & & & & 2 & & & & 2 & & 6 \\
\hline 15 & 2 & & & & & & & & 1 & 2 & & 5 \\
\hline 16 & 2 & & 1 & & & & & & 1 & 4 & & 8 \\
\hline 17 & 2 & & & & & & & & & & & 2 \\
\hline 18 & 1 & & 2 & & & & & & & 1 & & 4 \\
\hline 19 & 1 & & 2 & & & 2 & & & & 2 & & 7 \\
\hline 22 & & & & & & 1 & & & & 1 & & 2 \\
\hline 23 & 2 & & 1 & & & 1 & & & 1 & & & 5 \\
\hline 25 & 3 & & 2 & & 1 & 1 & 1 & & & 1 & & 9 \\
\hline 26 & 2 & & & 1 & & 1 & & & & 1 & & 5 \\
\hline 28 & 2 & & 2 & & & & 2 & 1 & 1 & 1 & & 9 \\
\hline 30 & 1 & & & 1 & & 1 & & & & 1 & 1 & 5 \\
\hline 31 & & & 2 & & 1 & & & 2 & & 4 & & 9 \\
\hline 33 & 2 & & 3 & & 1 & & & & & 2 & & 8 \\
\hline 37 & 1 & & 1 & & & 1 & & & 1 & 1 & & 5 \\
\hline Total & 34 & & 27 & 3 & 6 & 13 & 9 & 3 & 7 & 30 & 1 & 133 \\
\hline 25 & & & 70 & & & & 25 & & & 38 & \\
\hline
\end{tabular}


360 MetA, XLVIII, 3, 2003

TABLEAU $\mathrm{N}^{\circ} 7$

Aventures et mésaventures de la créativité chez les apprentis traducteurs

RÉSULTATS GÉNÉRAUX

\begin{tabular}{|c|c|c|c|c|c|c|c|c|c|c|c|c|}
\hline \multirow[t]{2}{*}{$\begin{array}{l}25 \\
\text { Élèves }\end{array}$} & \multicolumn{5}{|c|}{ Fautes de langue } & \multicolumn{3}{|c|}{$\begin{array}{c}\text { Fautes de } \\
\text { compréhension }\end{array}$} & \multicolumn{3}{|c|}{$\begin{array}{l}\text { Fautes de } \\
\text { technique }\end{array}$} & \multirow[t]{2}{*}{$\begin{array}{c}\text { Total } \\
\text { Fautes }\end{array}$} \\
\hline & Lex. & C.i. & Gram. & T.v. & Orth. & F.s. & N.s. & C.s. & Om. & Surt. & Soust. & \\
\hline $\begin{array}{l}\text { Fautes } \\
\text { répétées }\end{array}$ & 6 & & 3 & & 1 & 13 & & & 3 & & & \multirow[t]{2}{*}{26} \\
\hline Total & & & 10 & & & & 13 & & & 3 & & \\
\hline $\begin{array}{l}\text { Fautes } \\
\text { corrigées }\end{array}$ & 63 & 1 & 39 & 1 & 34 & 27 & 24 & 1 & 15 & 3 & & \multirow[t]{2}{*}{208} \\
\hline Total & & & 138 & & & & 52 & & & 18 & & \\
\hline $\begin{array}{l}\text { Fautes } \\
\text { nouvelles }\end{array}$ & 34 & & 27 & 3 & 6 & 13 & 9 & 3 & 7 & 30 & 1 & \multirow[t]{2}{*}{133} \\
\hline Total & \multicolumn{5}{|c|}{70} & \multicolumn{3}{|c|}{25} & \multicolumn{3}{|c|}{38} & \\
\hline
\end{tabular}

